\title{
Preliminary Analysis of Size Distribution of Objects Suspended in Normal Cerebrospinal Fluid in Case of Venticular Hydrocephalic Enlargement and Internal Hydrocephalus
}

\author{
W. StARON ${ }^{a}{ }^{*}$, L. KUBISz ${ }^{b}$ AND L. HerbOWSKI ${ }^{c}$ \\ ${ }^{a}$ Institute of Physics, University of Szczecin, Wielkopolska 15, 70-451 Szczecin, Poland \\ ${ }^{b}$ Department of Biophysics, University of Medical Sciences, Poznań, Poland \\ ${ }^{c}$ Public Provincial Hospital Complex in Szczecin, Szczecin, Poland
}

\begin{abstract}
The paper presents preliminary analysis of normal non-coloured cerebrospinal fluid obtained from patients diagnosed due to suspicion of cerebrospinal fluid malabsorption. According to the findings, the normal cerebrospinal fluid was classified into two groups: $A$ - with clinical diagnosis of ventricular hydrocephalic enlargement and $B$ - with clinical diagnosis of internal hydrocephalus. The analysis of microscopic pictures of normal cerebrospinal fluid in both groups according to numbers and sizes of suspended objects was performed with the aid of Eclipse 600 microscope (with magnification of $1200 \times$ ) working with a computer by a digital video camera. The authors observed that there is a significant difference in a shape of the distribution curve of objects' diameters between groups $A$ and $B$. The maximum number of objects in group $A$ was recorded within the range from 5 to $10 \mu \mathrm{m}$ in diameter size whereas in group $B$ the maximum was recorded within the range from 0.5 to $5 \mu$ m in diameter size.
\end{abstract}

PACS: 87.64.M-, 02.50.-r, 61.20.-p

\section{Introduction}

Body fluids, for which we include, inter alia, blood, urine, gastric juice, saliva or cerebrospinal fluid, due to the objects suspended in them form dispersion systems. Considering sizes of objects, the fluids may be graphically characterized by a size distribution curve of objects.

Microscopic examinations are still a gold standard in a laboratory analysis of cerebrospinal fluid to assess the small particles with diameters from $0.4 \mu \mathrm{m}$ [1]. Authors try to find the answer to the question whether in the chaos of particles in normal cerebrospinal fluid there is any regularity in a size distribution of suspended objects. The pioneering studies undertaken by the authors are completely different approach to the direct image analysis of cerebrospinal fluid and they have not been found in the world literature yet.

The paper is the next stage of research project on "Physico-chemical processes in cerebrospinal fluid obtained by puncture from patients diagnosed with the disorders of cerebrospinal fluid circulation" [2].

\section{Material and methodology}

Analyzed preparations of normal, non-coloured cerebrospinal fluid were obtained from the patients diagnosed due to suspicion of cerebrospinal malabsorption in the

* corresponding author; e-mail: staron@wmf.univ.szczecin.pl
Neurosurgery Department of Public Provincial Hospital Complex in Szczecin. The cerebrospinal fluid was taken during routine diagnostic procedures and treatment. The authors obtained the consent of Bioethical Committee of Regional Medical Chamber in Szczecin to conduct clinical trials. Based on tomography diagnosis patients were divided into two subgroups: $A$ (6 patients) - with clinical diagnosis of ventricular hydrocephalic enlargement and $B$ (13 patients) - with clinical diagnosis of internal hydrocephalus. The analysis of microscopic pictures of cerebrospinal fluid preparations in both groups according to numbers and sizes of suspended objects was performed with the aid of Eclipse 600 microscope working with a computer by a digital video camera.

Due to different shapes of suspended objects, it must be calculated an equivalent diameter of each object which allows one to compare the objects. The equivalent diameter $\mathrm{d} z$ was calculated with the use of the circle area formula comparing the total object specific area to a circle of the same area [3]. Statistical analysis was performed based on STATISTICA 7. Statistically significant differences were considered when their values $p<0.05$.

\section{Results}

The cerebrospinal fluid obtained from all the diagnosed patients was physiological both in the macroscopical (clear and transparent) and biochemical (normal protein and glucose level) examinations. The classical microscopic examination also showed its physiological parameters (cell content did not exceed 3 cells in $1 \mathrm{~mm}^{3}$ ). 
In analyzed samples of cerebrospinal fluid there were found 551 objects altogether: $A-104$ objects and $B-$ 447 objects. The equivalent diameters in each subgroup were ordered in growing series of $0.5 \mu \mathrm{m}$ wide.

The biggest equivalent object diameter found in subgroup $A$ was $21.5 \mu \mathrm{m}$ and the biggest equivalent object diameter found in subgroup $B$ was $15 \mu \mathrm{m}$. The smallest object in both groups was $1 \mu \mathrm{m}$ of the equivalent diameter size.

In both groups there were observed single objects of diameter size bigger than $12.5 \mu \mathrm{m}$. With the use of the Chauvenet criterion [4] the range of analyzed distributions was limited from $21.5 \mu \mathrm{m}$ to $12.5 \mu \mathrm{m}$ that allowed one to obtain clearer size distributions of object diameters. Based on measurement data and the Chauvenet criterion, percentage distributions of object diameters in both groups $A$ (Fig. 1) and $B$ (Fig. 2) were determined.

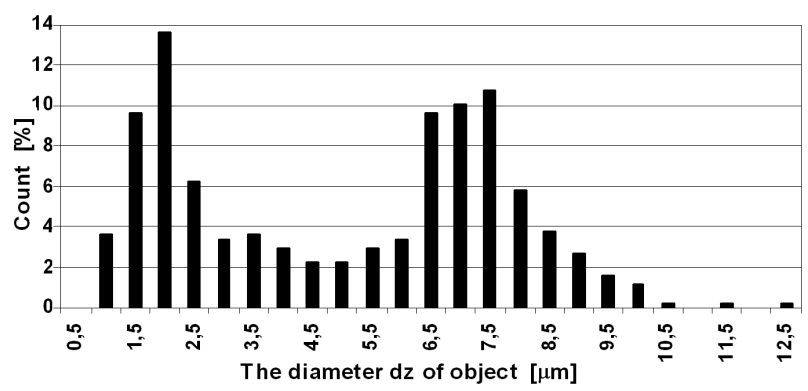

Fig. 1. Subgroup A. Percentage content of objects with equivalent diameter $\mathrm{d} z$ in normal cerebrospinal fluid.

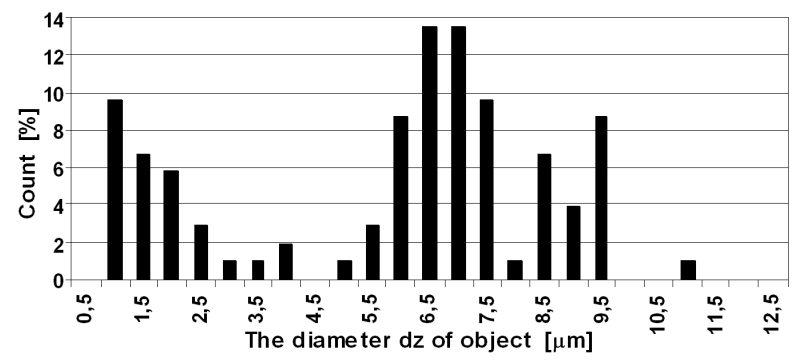

Fig. 2. Subgroup B. Percentage content of objects with equivalent diameter $\mathrm{d} z$ in normal cerebrospinal fluid.

Figures 1 and 2 show that the distributions are similar. In order to find out whether the object sizes have the same characteristics one used the Smirnov-Kolmogorov test [5]. At significance level $\alpha=0.05$, the $H_{0}$ hypothesis - "size distributions of objects are the same" — was rejected and alternative hypothesis — "size distributions of objects are not identical" - was accepted. It means that objects have different characteristics and come from different subpopulations.

To describe more precisely size distributions in both subgroups $A$ and $B$ one determined the following pa- rameters: median, mode and quartiles $Q 1, Q 2$, and $Q 3$. (Table).

TABLE

Parameters characterizing size distributions of objects in subgroups $A$ and $B$.

\begin{tabular}{c|c|c|c}
\hline \hline Parameter & Quartile $Q 1$ & Quartile $Q 2$ & Quartile $Q 3$ \\
\hline group $A$ & $2.76 \mu \mathrm{m}$ & $6.58 \mu \mathrm{m}$ & $7.61 \mu \mathrm{m}$ \\
group $B$ & $2.19 \mu \mathrm{m}$ & $5.70 \mu \mathrm{m}$ & $7.33 \mu \mathrm{m}$
\end{tabular}

Based on quartiles one determined coefficient of variation characterizing the inhomogeneity of object community [6]:

$$
V_{Q}=(Q 3-Q 1) /(Q 3+Q 1),
$$

where $Q 1$ - the first quartile, $Q 2$ - the second quartile, $Q 3$ - the third quartile. Coefficient of variation $V_{Q}$ in group $A$ is $46 \%$ and in group $B$ is $54 \%$.

Figures 1 and 2 show that the size distributions of subgroups $A$ and $B$ are bimodal. In both cases the modes are connected with the same classes of diameters. To compare the modes, the size distributions of diameters in each subgroup were divided into two parts. The first part applies to "small" objects within the range of 0.5 $4.5 \mu \mathrm{m}$ (Fig. 3) and the other one applies to "large" objects within the range of $5.0-12.5 \mu \mathrm{m}$ (Fig. 4).

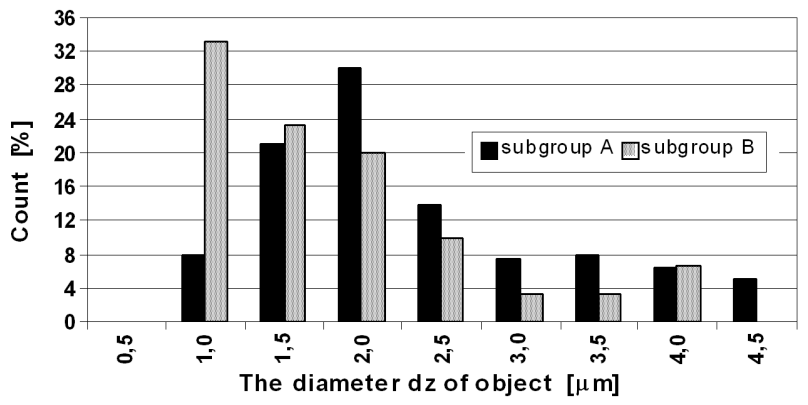

Fig. 3. Percentage content of objects with equivalent diameter $\mathrm{d} z$ (range of $0.5-4.5 \mu \mathrm{m}$ ) in normal cerebrospinal fluid.

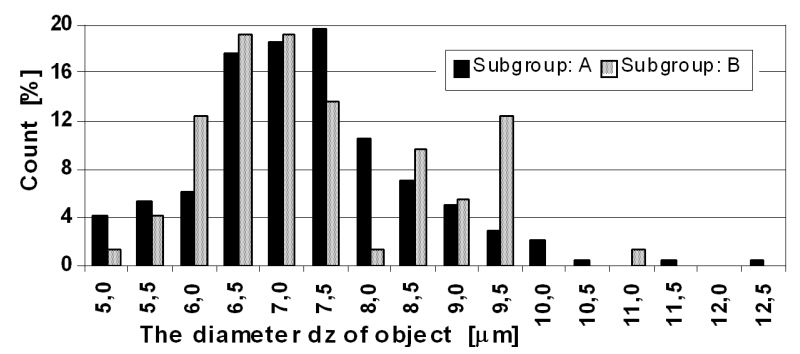

Fig. 4. Percentage content of objects with equivalent diameter $\mathrm{d} z$ (range of $5.0-12.5 \mu \mathrm{m}$ ) in normal cerebrospinal fluid. 
For "small" objects, the maximum is in the range of $2 \mu \mathrm{m}$ in subgroup $A$, and in the range of $1 \mu \mathrm{m}$ in subgroup $B$ (Fig. 3).

For "large" objects the maximum is in the range of $7.5 \mu \mathrm{m}$ in subgroup $A$, and in the range of 6.5 and $7 \mu \mathrm{m}$ in subgroup $B$ (Fig. 4).

\section{Discussion}

Properties of cerebrospinal fluid due to its complexity are described by various parameters. However, four of them: glucose level, protein level, chlorides level and cell content allow one to evaluate the cerebrospinal fluid as normal or pathological [7].

Microscopic examinations indicate presence of short and long size distributions of objects suspended in normal non-coloured cerebrospinal fluid. Cumulative size distribution of objects in examining material obtained from patients diagnosed with disorders of cerebrospinal fluid circulation has bimodal shape. According to the laws of statistics, bimodal distribution suggests that the test group is inhomogeneous, whereas the SmirnovKolmogorov test confirmed that the objects have different characteristics and come from different subpopulations.

For the selected subgroups $A$ and $B$ graphs of the size distributions of equivalent diameters $\mathrm{d} z$ were plotted. In both cases the distributions are bimodal. In the subgroup $A$ the maximum is recorded in the ranges of $2 \mu \mathrm{m}$ and $7.5 \mu \mathrm{m}$ and it is, respectively, $30.2 \%$ and $19.7 \%$. In the subgroup $B$ the maximum is recorded in the ranges of $1 \mu \mathrm{m}$ and $6.5 \mu \mathrm{m}$ and it is, respectively, $33.3 \%$ and $19.2 \%$.

On the basis of graphical presentation (Fig. 1 and Fig. 2) and results of positioning measurements of a size distribution it is concluded that the size distributions of objects are asymmetrical.

To determine the direction of asymmetry, the coefficient of asymmetry was calculated as follows [6]:

$$
X=(Q 3-Q 2)-(Q 2-Q 1),
$$

where $Q 1$ - the first quartile, $Q 2$ - the second quartile,
Q3 - the third quartile. In both groups the distributions show a left-sided asymmetry which is stronger in group $A$ for which the coefficient of asymmetry is $X=0.57$. For group $B$ the coefficient of asymmetry is $X=0.36$.

\section{Conclusions}

1. Based on statistical analysis of objects parameters and clinical procedures we may distinguish two subgroups.

2. The subgroups $A$ and $B$ differ in parameters describing size distributions of objects.

3. On the basis of size distributions of objects suspended in cerebrospinal fluid it is possible to diagnose internal hydrocephalus or ventricular hydrocephalic enlargement.

4. The studies show that the microscopic method may be useful for diagnostically difficult differentiation of disorders of cerebrospinal fluid circulation.

\section{References}

[1] J.T. Van Hacker, J.R. Delanghe, M.R. Langlois, Y.E. Taes, M.L. de Buyzere, A.G. Verstraete, Clin. Chem. 47, 556 (2001).

[2] W. Staroń, L. Herbowski, Acta Phys. Pol. A 118, 1 (2010).

[3] K.W. Zieliński, M. Strzelecki, Computer Analysis of Biomedical Picture, PWN, Warsaw 2002 (in Polish).

[4] J.R. Taylor, An Introduction to Error Analysis, PWN, Warsaw 1995 (in Polish).

[5] C. Watała, Biostatistics, $\alpha$-Medica Press, Bielsko-Biała 2002 (in Polish).

[6] M. Sobczak, Statistics, PWN, Warsaw 2007 (in Polish).

[7] R. Caquet, 250 Laboratory Tests. Requirements and Interpretation, PZWL, Warsaw 2007 (in Polish). 